VOL. 60 (1999) [227-238]

\title{
SUBGROUP COVERINGS OF SOME LINEAR GROUPS
}

\author{
R.A. BRYCE, V. FEDri and L. SERENA \\ Dedicated to Bernhard Neumann on his 90th birthday.
}

\begin{abstract}
A cover for a group is a collection of proper subgroups whose union is the whole group. A cover is minimal if no other cover contains fewer members. We term minimised a minimal cover with the property that substituting for a member of the cover by a proper subgroup of that member produces a collection which is no longer a cover. We here describe the minimised covers for the groups $\mathrm{GL}_{2}(q), \mathrm{SL}_{2}(q), \mathrm{PSL}_{2}(q)$ and $\mathrm{PGL}_{2}(q)$.
\end{abstract}

\section{INTRODUCTION}

Let $G$ be a group. A cover of $G$ is a collection $\mathcal{A}=\left\{A_{i}: 1 \leqslant i \leqslant n\right\}$ of proper subgroups of $G$ whose union is $G$. The cover $\mathcal{A}$ is irredundant if no proper sub-collection is also a cover; and minimal if no cover of $G$ has fewer than $n$ members. In this minimal case we write $\sigma(G)=n$.

Covers of groups have been studied by many authors. For example Neumann [5] shows that the intersection of the members of an irredundant cover with $n$ members has index bounded by a function of $n$. Tomkinson [8] improved this bound. Minimal covers seem to have been introduced by Cohn [1]: and Tomkinson [9] showed that, for a finite soluble group $G, \sigma(G)$ is $p^{\alpha}+1$ where $p^{\alpha}$ is the size of the smallest chief factor of $G$ with multiple complements. He confirmed a conjecture of Cohn $[1]$ that $\sigma(G)=7$ for no group $G$. His proof suggests that investigating minimal covers of insoluble groups might be of interest. Here we make a small beginning by looking at the groups $\mathrm{GL}_{2}(q)$, $\mathrm{SL}_{2}(q), \mathrm{PSL}_{2}(q)$ and $\mathrm{PGL}_{2}(q)$ (Theorem 3.5). We find $\sigma$ for these groups and, more to the point, we give a description of all the minimal covers which are minimised in a sense to be described below (Theorem 4.4).

\section{Received 28th April, 1999}

Following Prof. Fedri's death at Easter 1998 the other two authors have produced this account of our preliminary investigations into coverings of the general linear and related groups.

The first author thanks the Dipartimento di Matematica "Ulisse Dini" of the Università degli Studi di Firenze for its hospitality and generous financial support in October and November 1997.

Copyright Clearance Centre, Inc. Serial-fee code: 0004-9727/99 \$A2.00+0.00. 


\section{QUOTATIONS AND NOTATION}

In what follows $G$ will always denote one of the groups $\mathrm{GL}_{2}(q), \mathrm{SL}_{2}(q), \mathrm{PSL}_{2}(q)$ and $\mathrm{PGL}_{2}(q)$ where $q=p^{\alpha}$ is a prime power, always at least 4 except in Section 5. In contexts where the symbol $G$ is unquantified it may be interpreted as any one of the four groups. Of course, when $q$ is even, the four groups comprise just two isomorphism classes represented by $\mathrm{GL}_{2}(q)$ and $\mathrm{SL}_{2}(q)$. We associate a parameter $\varepsilon$ with $G$ as follows: $\varepsilon=1$ except in the single case when $q$ is odd and $G$ is $\operatorname{PSL}_{2}(q)$ when $\varepsilon=1 / 2$. Let $V$ be the space on which $\mathrm{GL}_{2}(q)$ acts so that all four groups have a natural action on the projective line $\mathcal{P}(1, q)$ thought of as the set of one-dimensional subspaces of $V$. It will be convenient to assign a labelling $\{1,2, \ldots, q+1\}$ to the points of $\mathcal{P}(1, q)$; this assignment is arbitrary, but then fixed. The stabiliser in $G$ of point $i$ we denote by $P_{i}$ and its unipotent radical by $N_{i}$; note that $N_{i}=O_{p}\left(P_{i}\right)$, and that $N_{i}$ is a Sylow $p$-subgroup of $G$. It is well-known that $P_{i}$ is maximal in $G$.

The structure of a point stabiliser $P_{i}$ is well-known. We may write $P_{i}=F_{i} Z$ where $Z$ is the centre of $G$, and $F_{i} Z / Z$ is a Frobenius group of order $\varepsilon q(q-1)$ with Frobenius kernel $N_{i} Z / Z$. Of course, except for $\mathrm{GL}_{2}(q), Z$ in the product $F_{i} Z$ is redundant; and, in the case of $\mathrm{GL}_{2}(q)$, the product $F_{i} Z$ is direct. $F_{i}$ is not unique when $G=\mathrm{GL}_{2}(q)$, but each choice for it is a supplement for $\mathrm{SL}_{2}(q)$ in $G$.

Let us write $K_{i j}:=F_{i} \cap P_{j}(i \neq j)$. Then

$$
\left|K_{i j}\right|=\varepsilon(q-1)
$$

and

$$
\left|K_{i j} \cap Z\right|= \begin{cases}2, & G=\mathrm{SL}_{2}(q)(q \text { odd }) \\ 1, & \text { otherwise }\end{cases}
$$

Moreover

$$
K_{i j} Z=K_{j i} Z=P_{i} \cap P_{j}(i \neq j) .
$$

$K_{i j}$ is an example of an eccentric cycle in $P_{i} \cap P_{j}$, namely a cyclic supplement for $Z$ in $P_{i} \cap P_{j}$. Every eccentric cycle arises as $F_{i} \cap P_{j}$ for some choice of $F_{i}$. In $\mathrm{GL}_{2}(q)$ eccentric cycles are complements for $Z$ in $P_{i} \cap P_{j}$, and distinct eccentric cycles generate $P_{i} \cap P_{j}$. $G L_{2}(q)=\mathrm{SL}_{2}(q) K_{i j}$ for every eccentric cycle $K_{i j}$.

A Singer cycle of $\mathrm{GL}_{2}(q)$ is a cyclic subgroup of order $q^{2}-1$. Every Singer cycle acts irreducibly on $V$. The Singer cycles therefore form a single conjugacy class of $\mathrm{GL}_{2}(q)$, and Singer cycles are self-centralising; in particular $Z$ is in every Singer cycle. If $S$ is a Singer cycle of $\mathrm{GL}_{2}(q)$ we shall term $S \cap \mathrm{SL}_{2}(q),\left(S \cap \mathrm{SL}_{2}(q)\right) /\left(Z \cap \mathrm{SL}_{2}(q)\right)$ and $S / Z$ Singer cycles of $\mathrm{SL}_{2}(q), \mathrm{PSL}_{2}(q)$ and $\mathrm{PGL}_{2}(q)$ respectively. By Huppert $[3,7.3]$ $\left|S \cap \mathrm{SL}_{2}(q)\right|=q+1$ and $\mathrm{GL}_{2}(q)=\mathrm{SL}_{2}(q) S$. Also, using this same result from Huppert, whenever $D$ is a Singer normaliser in $\mathrm{GL}_{2}(q)$, then $D \cap \mathrm{SL}_{2}(q),\left(D \cap \mathrm{SL}_{2}(q)\right) /\left(Z \cap \mathrm{SL}_{2}(q)\right)$ and $D / Z$ are Singer normalisers in $\mathrm{SL}_{2}(q), \mathrm{PSL}_{2}(q)$ and $\mathrm{PGL}_{2}(q)$ respectively. The same 
result shows that the Singer cycles of $\mathrm{SL}_{2}(q)$ are a single conjugacy class of $\mathrm{SL}_{2}(q)$; that the order of the normaliser of a Singer cycle $S$ is $2|S|$; and that the number of Singer cycles is $s:=q(q-1) / 2$ whichever of the four groups $G$ might be.

Now it is easy to see that, whenever $S$ is a Singer cycle and $1 \leqslant i \leqslant q+1$,

$$
G=N_{G}(S) P_{i}
$$

and

$$
\left|P_{i} \cap N_{G}(S)\right|=\left\{\begin{aligned}
2(q-1), & G=\mathrm{GL}_{2}(q) \\
2, & G=\mathrm{SL}_{2}(q) \\
2, & G=\mathrm{PGL}_{2}(q) \\
1, & G=\mathrm{PSL}_{2}(q)(q \text { odd }) .
\end{aligned}\right.
$$

We denote the set of Singer subgroups of $G$ by $\Sigma$ and the set of their normalisers in $G$ by $\bar{\Sigma}$. In the same spirit if $\Sigma_{0} \subseteq \Sigma$ then $\bar{\Sigma}_{0}$ denotes the set of the normalisers of the members of $\Sigma_{0}$.

We denote by $\Pi$ the set of all point stabilisers in $G$. It will be convenient to write $\Pi_{i}$ for $\Pi \backslash\left\{P_{i}\right\}$ and $\Pi_{i j}$ for $\Pi \backslash\left\{P_{i}, P_{j}\right\}(i \neq j)$.

It will be useful to make the following definition in the case that $q$ is odd:

$$
M:= \begin{cases}\mathrm{SL}_{2}(q) Z, & G=\mathrm{GL}_{2}(q)(q \text { odd }) \\ \mathrm{SL}_{2}(q) Z / Z, & G=\mathrm{PGL}_{2}(q)(q \text { odd }) .\end{cases}
$$

Note that $M$ is a maximal subgroup of $G$.

We rely throughout this article on Dickson's list of subgroups of $\mathrm{PSL}_{2}(q)$ as given in Huppert (8.27 in [3]) and, to a lesser extent where convenient, on the Atlas [2]. Observe that in Huppert's 8.27 (7) there is a misprint: the orders of the subgroups involved are divisors of $\varepsilon q(q-1)$.

\section{Preliminary Results}

Here we build up the results we need for the description of the minimised covers in the next section. One of the results we obtain here is the value of $\sigma(G)$ for all but a few small cases. These missing cases are treated in Section 5.

Lemma 3.1. The union $\Sigma \cup \Pi$ is a cover for $G$. When $q$ is even $\bar{\Sigma} \cup \Pi_{i}$ is a cover of $G$ for each $i \in\{1,2, \ldots, q+1\}$.

Proof: An element of $\mathrm{GL}_{2}(q)$ acting without a fixed point in $\mathcal{P}(1, q)$ is contained in a Singer cycle. Hence $\Sigma \cup \Pi$ is a cover for $G$.

The second statement comes from the fact that

$$
P_{i}=N_{i} Z \cup \bigcup_{j \neq i}\left(P_{i} \cap P_{j}\right)
$$

and that, by (4), for $q$ even, each element of $N_{i} Z$ is contained in some Singer normaliser. 
Corollary 3.2 .

$$
\sigma(G) \leqslant \begin{cases}\frac{1}{2} q(q+1), & q \text { even } \\ \frac{1}{2} q(q+1)+1, & q \text { odd } .\end{cases}
$$

Lemma 3.3. For $q \geqslant 4$ the normaliser of a Singer cycle $S$ is the unique maximal subgroup of $G$ containing $S$ except when $G=(P) S L_{2}(q)$ and $q=5,7,9$.

Proof: When $G=(P) \mathrm{GL}_{2}(q)$ this follows from a special case of Kantor [4]. When $G=(P) \mathrm{SL}_{2}(q)$ the result follows by reference to Dickson's list. A maximal subgroup $U$ of $\mathrm{SL}_{2}(q)$ containing a Singer cycle $S$ satisfies $(q+1)|| U \mid$; and $Z \subseteq U$ because $\mathrm{SL}_{2}(q)$ is perfect. Then $\varepsilon(q+1)|| U / Z \mid$ and it is straight-forward to check that, unless $q$ is odd and $q \leqslant 9, U / Z$ is the normaliser of $Z$.

LEMMA 3.4 .

1. Let $G=\mathrm{GL}_{2}(q)$. Then
(a) $\left\langle N_{i}, N\right\rangle=S L_{2}(q) \quad\left(1 \neq N \subseteq N_{j}, i \neq j, q \geqslant 4\right)$
(b) $\left\langle K_{i j}, N_{k}\right\rangle=G \quad(i \neq k \neq j \neq i, q \geqslant 4)$
(c) $\left\langle K_{i j}, K_{k \ell}\right\rangle=G \quad(\{i, j\} \cap\{k, \ell\}=\emptyset, q \geqslant 7)$;

2. When $G=S L_{2}(q) 1(\mathrm{~b})$ holds for $q \geqslant 4$; and 1(c) holds for $q \geqslant 4$ but $q \neq 5,7,9,11$.

Proof: Let $G$ be either $\mathrm{GL}_{2}(q)$ or $\mathrm{SL}_{2}(q)$. Now $K_{i j}$ acts on $\mathcal{P}(1, q)$ with orbit lengths 1,1 and $q-1$; and $N_{i}$ acts with orbit lengths 1 and $q$. Hence, under the conditions imposed, $\left\langle N_{i}, N\right\rangle,\left\langle K_{i j}, N_{k}\right\rangle$ and $\left\langle K_{i j}, K_{k \ell}\right\rangle$ act transitively on $\mathcal{P}(1, q)$. Therefore $q+1$ divides the order of each.

1(a) Now $H:=\left\langle N_{i}, N\right\rangle \subseteq \mathrm{SL}_{2}(q)$; and $q(q+1)|| H \mid$. If $H \neq \mathrm{SL}_{2}(q)$ then $H Z / Z$ is a proper subgroup of $\operatorname{PSL}_{2}(q)$ and $\varepsilon q(q+1)|| H Z / Z \mid$. From Dickson's list [3, 8.27] the only proper subgroups of $\mathrm{PSL}_{2}(q)$ whose orders are divisible by $q$ are contained in a Frobenius group of order $\varepsilon q(q-1)$, which is not divisible by $\varepsilon q(q+1)$, or are in subgroups isomorphic to $A_{4}, S_{4}$ or $A_{5}$; an easy calculation shows that, for $q \geqslant 4$, none of these is possible. This contradiction means that $H\left(Z \cap \mathrm{SL}_{2}(q)\right)=\mathrm{SL}_{2}(q)$ and so $H=\mathrm{SL}_{2}(q)$ since $\mathrm{SL}_{2}(q)$ is perfect.

1(b) Let $H:=\left\langle K_{i j}, N_{k}\right\rangle$ and $H_{0}:=H \cap \mathrm{SL}_{2}(q)$ so that $H=K_{i j} H_{0}$. Also let $Z_{0}:=Z \cap \mathrm{SL}_{2}(q)$. Suppose $H_{0} \neq \mathrm{SL}_{2}(q)$ and let $U_{0}$ be a maximal subgroup of $\mathrm{SL}_{2}(q)$ containing $H_{0}$; since $\mathrm{SL}_{2}(q)$ is perfect $Z_{0} \subseteq U_{0}$. Now $q\left(q^{2}-1\right)|| H \mid$ or $q\left(q^{2}-1\right) / 2$ according as $q$ is even or odd, so either $\varepsilon q(q+1)|| U_{0} / Z_{0} \mid(q \equiv 1(\bmod 4))$ or $\varepsilon^{2} q(q+1) \mid$ $\left|U_{0} / Z_{0}\right|(q \equiv 3(\bmod 4))$. Much as in the last paragraph we conclude that, for $q \geqslant 4$, $H_{0} / Z_{0}=\mathrm{PSL}_{2}(q)$ whence $H_{0}=\mathrm{SL}_{2}(q)$, and so $H=G$, a contradiction. 
1(c) Let $H=\left\langle K_{i j}, K_{k \ell}\right\rangle$. Define $H_{0}:=H Z \cap \mathrm{SL}_{2}(q)$ so that $H Z=K_{i j} H_{0}$. Write $Z_{0}:=Z \cap \mathrm{SL}_{2}(q) . K_{i j} \times Z \subseteq H Z$ so $\left(q^{2}-1\right)(q-1)|| H Z \mid$ or $\left(q^{2}-1\right)(q-1) / 4$ according as $q$ is even or odd, whence $\varepsilon^{3}\left(q^{2}-1\right)|| H_{0} / Z_{0} \mid$.

Suppose that $H Z \neq G$ so that $H_{0} \neq \mathrm{SL}_{2}(q)$. We produce a contradiction to this assumption using Dickson's list of subgroup of $\mathrm{PSL}_{2}(q)$.

We show, first of all, that $H_{0} / Z_{0}$ is not isomorphic to one of $A_{4}, S_{4}, A_{5}$. Since $q \geqslant 7$, $H_{0} / Z_{0}$ does not in these cases have an automorphism of order $q-1$ (note that $H_{0} / Z_{0} \cong A_{5}$ does not arise when $q=7$ ). Hence $K_{i j}$ does not act faithfully on $H_{0} / Z_{0}$, so there is a nonidentity (non-central) element $y \in K_{i j}$ satisfying $\left[H_{0}, y\right] \subseteq Z_{0}$. Now the function $H_{0} \rightarrow Z_{0}$ defined by $x \mapsto[x, y]$ is a homomorphism, and so $\left[O^{2}\left(H_{0}\right), y\right]=1$ since $\left|Z_{0}\right|=2$. Since $O^{2}\left(H_{0}\right)$ is an irreducible subgroup of $G$ its centraliser in $G$ is cyclic. However $\langle y\rangle Z$ is not cyclic, a contradiction in each of the three cases.

For $q \geqslant 7$, the other possibilities for $H_{0} / Z_{0}$ are: cyclic of order dividing $\varepsilon(q \pm 1)$, dihedral of order dividing $2 \varepsilon(q \pm 1)$, subgroups of Frobenius groups of order $\varepsilon q(q-1)$, $\mathrm{PSL}_{2}\left(p^{m}\right)(m \mid \alpha)$ and $\mathrm{PGL}_{2}\left(p^{m}\right)(2 m \mid \alpha)$. By checking these for divisibility of their orders by $\varepsilon^{3}\left(q^{2}-1\right)$ we eliminate all but the cases $q=7$ and $H_{0} / Z_{0} \cong S_{3}$, and $q=9$ and $H_{0} / Z_{0} \cong D_{10}$. The second case is eliminated exactly as in the last paragraph. In the first case $H_{0}$ is a non-Abelian semi-direct product of a cyclic group of order 3 by a cyclic group of order 4 . Now $V$ is irreducible for $H_{0}$, in fact absolutely irreducible since its dimension is prime. Therefore $C_{G}\left(H_{0}\right)=Z$. Also the automorphism group of $H_{0}$ has unique Sylow subgroup of order 3 . Let $y_{1}$ and $y_{2}$ be elements of order 3 in $K_{i j}$ and $K_{k \ell}$ respectively. It follows that $y_{1} y_{2}^{ \pm 1} \in C_{G}\left(H_{0}\right)=Z$. However this contradicts the fact that $y_{1}, y_{2}$ fix different points.

We have shown, therefore, that $H Z=G$. Hence $\mathrm{SL}_{2}(q)=G^{\prime} \subseteq H$ so $G=$ $\mathrm{SL}_{2}(q) K_{i j} \subseteq H$ whence $H=G$.

This concludes the proof of $1(c)$.

(2) If $H:=\left\langle K_{i j}, N_{k}\right\rangle \neq G$ we see that $\varepsilon^{2} q\left(q^{2}-1\right)|| H / Z \mid$. This is dismissed, for $q \geqslant 4$, much as in the proof of $1(\mathrm{a})$ above.

If $H:=\left\langle K_{i j}, K_{k \ell}\right\rangle \neq \mathrm{SL}_{2}(q)=G$ we deduce that $\varepsilon^{2}\left(q^{2}-1\right)|| H / Z \mid$. Checking through Dickson's list yields that $q$ is odd and $q \leqslant 11$.

It is not difficult to see that the proscription $q \neq 5,7,9,11$ is necessary in the second part of (2) of this lemma.

Before stating the next theorem we introduce some convenient notation. A poset may be defined on the set of collections of subgroups of a group $H$ as follows. If $\mathcal{A}=$ $\left\{A_{i}: 1 \leqslant i \leqslant m\right\}$ and $\mathcal{B}=\left\{B_{i}: 1 \leqslant i \leqslant n\right\}$ are collections of subgroups of $H$ we say $\mathcal{A} \preceq \mathcal{B}$ if for some one-to-one function $f:\{1,2, \ldots, m\} \rightarrow\{1,2, \ldots, n\}$ we have $A_{i} \subseteq B_{f(i)}(1 \leqslant i \leqslant m)$. The poset so defined we denote by $\Delta_{H}$. 
THEOREM 3.5. Let $q \geqslant 4$. Then

$$
\sigma(G)= \begin{cases}\frac{1}{2} q(q+1), & q \text { even } \\ \frac{1}{2} q(q+1)+1, & q \text { odd }\end{cases}
$$

provided either $G=(P) \mathrm{GL}_{2}(q)$, or $G=(P) S L_{2}(q)$ and $q \neq 5,7,9$.

Proof: Let $\mathcal{A}$ be a minimal cover for $G$. If each member of $\mathcal{A}$ is replaced by a maximal subgroup of $G$ containing it the resulting set is still a (minimal) cover for $G$. Hence we may suppose that $\mathcal{A}$ consists of maximal subgroups $A_{i}(1 \leqslant i \leqslant \sigma(G))$ of $G$.

Now each Singer cycle $S$ is in some member of $\mathcal{A}$. Since, by Lemma 3.3, the unique maximal subgroup containing a Singer cycle is its normaliser, which has order $2|S|$, it follows that different Singer cycles are in different members of $\mathcal{A}$, let us say in $A_{k}(1 \leqslant$ $k \leqslant s=q(q-1) / 2)$.

Using Lemma 3.41 (a) we see that distinct $F_{i}$ generate $G$, so do not belong to the same member of $\mathcal{A}$. Hence, if $q$ is odd and $\left\{F_{i}\right\} \preceq \mathcal{A}(1 \leqslant i \leqslant q+1)$, or if $q$ is even and $\left\{F_{i}\right\} \preceq \mathcal{A}$ for all but one $i$, we are done by Corollary 3.2. Suppose, for some $i$, that

$$
\left\{F_{i}\right\} \npreceq \mathcal{A} .
$$

None of the subgroups $K_{i j}(j \neq i)$ is in $A_{k}(1 \leqslant k \leqslant s)$; for, under our conditions, a Singer normaliser contains no eccentric cycle of order $\varepsilon(q-1)$. Moreover no two are in the same member of $\mathcal{A}$ since two together generate $F_{i}$. Hence

$$
K_{i j} \subseteq A_{s+j}(j \neq i)
$$

say. If $q$ is even then $n \geqslant q(q-1) / 2+q=q(q+1) / 2$ and we are done, by Corollary 3.2. If $q$ is odd then

$$
N_{i} \cap A_{k}=1(k \neq s+i) .
$$

This is because $q$ is co-prime to the order of the Singer normalisers on the one hand and, if $N_{i} \cap A_{s+k} \neq 1$ for some $k \neq i$, then $F_{i}=\left\langle K_{i k}, N_{i} \cap A_{s+k}\right\rangle \subseteq A_{s+k}$, a contradiction, on the other. Therefore $N_{i}$ intersects another member of $\mathcal{A}$ non-trivially. Hence,

$$
n \geqslant \frac{1}{2} q(q-1)+q+1
$$

so, by Corollary 3.2 again, we are done.

\section{Minimised COVERS}

The subposet of $\Delta_{H}$ consisting of minimal covers for $H$ we denote $\Gamma_{H}$. Our aim now is to describe the minimal elements of $\Gamma_{G}$ : these are the minimised covers of the heading. of this section. The relative conditions imposed on $q$ and $G$ in the lemma we now state are designed to ensure that the hypotheses of Lemmas 3.3, 3.4 hold. 
LEMMA 4.1. Let $q \geqslant 7$ in the case that $G=(P) \mathrm{GL}_{2}(q)$, and $q \geqslant 4$ but $q \neq$ $5,7,9,11$ when $G=(P) S L_{2}(q)$. Also let $\mathcal{A}$ be a minimal cover of $G$. Then, for some integers $i, j$ satisfying $1 \leqslant i<j \leqslant q+1$,

$$
\Sigma \cup \Pi_{i j} \preceq \mathcal{A} \text {. }
$$

Proof: As before write $\mathcal{A}:=\left\{A_{i}: 1 \leqslant i \leqslant n\right\}$ where $n=\sigma(G)$. It is clear that $\Sigma \preceq \mathcal{A}$, say the subgroups $A_{i}(1 \leqslant i \leqslant s)$ contain the Singer cycles.

Using Lemma 3.41 (a) no two $F_{i}$ s are in the one member of $\mathcal{A}$ since, under our hypotheses, they generate $G$. Suppose that two $F_{i}$ s do not belong to members of $\mathcal{A}$; say, for notational simplicity, that $\left\{F_{1}\right\} \npreceq \mathcal{A}$ and $\left\{F_{2}\right\} \npreceq \mathcal{A}$. Now the $q$ cyclic subgroups $K_{1 k}(1<k \leqslant q+1)$ do not belong to any $A_{\ell}(1 \leqslant \ell \leqslant s)$, and no two belong to the same member of $\mathcal{A}$ since together they generate $F_{1}$. Let us say that

$$
K_{1 k} \subseteq A_{s+k}(1<k \leqslant q+1)
$$

When $q$ is odd, moreover,

$$
N_{1} \subseteq A_{s+1}
$$

because the argument following (5) shows that $N_{1}$ intersects every other member of $\mathcal{A}$ trivially.

The cyclic subgroups $K_{2 k}$ do not belong to $A_{i}(1 \leqslant i \leqslant s)$; and, from Lemma 3.4 $1(c), 2, K_{2 k} \nsubseteq A_{s+\ell}(\ell \neq k)$. Hence $K_{2 k} \subseteq A_{s+k}$. Therefore,

$$
F_{k}=\left\langle K_{1 k}, K_{2 k}\right\rangle \subseteq A_{s+k}(1 \neq k \neq 2) .
$$

Indeed this remains true whatever eccentric cycle in $K_{2 k} Z$ we use in place of $K_{2 k}$, so $Z \subseteq A_{s+k}$ whence $P_{k}=F_{k} Z \subseteq A_{s+k}$. This confirms the claim of the Lemma in the case that two $F_{i}$ s are not contained in members of $\mathcal{A}$.

Suppose just one $F_{i}$, say $F_{1}$, is not in a member of $\mathcal{A}$. Then we may suppose that

$$
F_{j} \subseteq A_{s+j}(1<j \leqslant q+1) .
$$

If $q$ is odd then, as above, $N_{1} \subseteq A_{s+1}$. In any case, as above, every eccentric cycle in $K_{1 \ell} Z$ is in $A_{s+\ell}(1<\ell \leqslant q+1)$. It follows that $P_{\ell} \subseteq A_{s+\ell}(1<\ell \leqslant q+1)$. This concludes the proof in this case.

Suppose finally that every $F_{i}$ is contained in some member of $\mathcal{A}$ (so that $q$ is odd), say

$$
F_{i} \subseteq A_{s+i}(1 \leqslant i \leqslant q+1)
$$

Now

$$
N_{i} Z \cap A_{j} \subseteq Z(j \neq s+i)
$$


on account of Lemma 3.41 (a) and the fact that Singer normalisers have order co-prime to $q$ (recall that $q$ is odd here). Hence $N_{i} Z \subseteq A_{s+i}$ and so $P_{i}=F_{i} Z \subseteq A_{s+i}$. This again confirms the conclusion of the Lemma.

We now begin a description of the minimised covers, always under the hypotheses of Lemma 4.1 so that Lemmas $3.3,3.4$ are in force. To this end we first of all derive necessary conditions on a cover $\mathcal{A}$ of $G$ in order that it be minimal.

At most two point stabilisers are not in $\mathcal{A}$, by Lemma 4.1. Suppose that $P_{i}$ and $P_{j}$ are not in $\mathcal{A}$. First consider the case when $q$ is odd. Now, by Lemma $3.41(\mathrm{a})$, and the fact that $p$ does not divide the order of a Singer normaliser, $N_{i}$ and $N_{j}$ each intersect trivially the members of $\mathcal{A}$ containing the subgroups in $\Sigma \cup \Pi_{i j}$. By Theorem 3.5 there are two other members of $\mathcal{A}$, say $X, Y$, and

$$
N_{i} Z \cap A=N_{j} Z \cap A=Z(A \in \mathcal{A}, X \neq A \neq Y) \text {. }
$$

It follows that

$$
N_{i} Z=Z \cup\left(N_{i} Z \cap X\right) \cup\left(N_{i} Z \cap Y\right) \text {. }
$$

This union is redundant. If not, as is well-known (it is an old result of Scorza [6]),

$$
\begin{aligned}
4=\left|N_{i} Z: Z \cap\left(N_{i} Z \cap X\right) \cap\left(N_{i} Z \cap Y\right)\right| & =\left|N_{i} Z: Z \cap X \cap Y\right| \\
& =\left|N_{i} Z: Z\right||Z: Z \cap X \cap Y|,
\end{aligned}
$$

a contradiction since $\left|N_{i} Z: Z\right|=\left|N_{i}\right|$ is odd. Hence $N_{i} Z$ is contained in $X$ or $Y$. Similarly $N_{j} Z$ is in $X$ or $Y$. Therefore, changing names if necessary, either

(a) $M=\left\langle N_{i}, N_{j}\right\rangle Z=X$, and in this case $G=(P) \mathrm{GL}_{2}(q)$; or

(b) $N_{i} Z \subseteq X$ and $N_{j} Z \subseteq Y$.

Now $K_{i j}$ must be in $X$ or $Y$ : using Lemma 3.4 and the fact that, under our hypotheses, no Singer normaliser contains an eccentric cycle. Case (b) therefore does not arise since $P_{i}, P_{j}$ are not in $\mathcal{A}$. In case (a) it must be that every eccentric cycle from $P_{i} \cap P_{j}$ is in $Y$.

It follows that, when $q$ is odd, and $\mathcal{A}$ lacks two point stabilisers $P_{i}$ and $P_{j}, G=$ $(P) \mathrm{GL}_{2}(q), X=M$ and $K_{i j} Z \subseteq Y$.

When $q$ is even, and $\mathcal{A}$ lacks two point stabilisers, $P_{i}$ and $P_{j}$ say, then there is a unique member $X$ of $\mathcal{A}$ other than those containing the members of $\Sigma \cup \Pi_{i j}$, by Lemma 3.5. By Lemma 3.4, $K_{i j} Z \subseteq X$. Now $X \cap N_{i}=X \cap N_{j}=1$, and $N_{i}$ and $N_{j}$ intersect trivially the point stabilisers $P_{k}(i \neq k \neq j)$. Hence $N_{i} \cap W \neq 1$ for some $W \in \mathcal{A}$ containing a Singer cycle. Therefore $W \in \bar{\Sigma}$. However no two involutions in a Singer normaliser commute, so $\left|W \cap N_{i}\right|=2$. It follows that, for each non-trivial $v \in N_{i}$, there is a Singer normaliser $\bar{S}_{v} \in \mathcal{A}$ such that $\bar{S}_{v} \cap N_{i}=\langle v\rangle$. Similarly for each non-trivial $v \in N_{j}$ there exits $T_{v} \in \Sigma$ such that $\overline{T_{v}} \in \mathcal{A}$ and $\overline{T_{v}} \cap N_{j}=\langle v\rangle$.

Let us write, for $1 \leqslant k \leqslant q+1$ and for $1 \neq v \in N_{k}$,

$$
\Sigma_{k, v}:=\left\{S \in \Sigma: \bar{S} \cap N_{k}=\langle v\rangle\right\}
$$


Observe that, by (4), $N_{G}(S) \cap N_{i} \neq 1(S \in \Sigma, 1 \leqslant i \leqslant q+1)$. Then, for each $k \in$ $\{1,2, \ldots, q+1\}, \Sigma=\bigcup_{1 \neq v \in N_{k}} \Sigma_{k, v}$; conjugation by $N_{k}$ permutes $\Sigma_{k, v}$ transitively with kernel $\langle v\rangle$; and conjugation action by $P_{k}$ permutes the set $\left\{\Sigma_{k, v}: 1 \neq v \in N_{k}\right\}$ transitively. Let us call a subset $\Sigma_{k}$ of $\Sigma$ a $k$-transversal if $\left|\Sigma_{k} \cap \Sigma_{k, v}\right|=1\left(1 \neq v \in N_{k}\right)$.

What we showed in the penultimate paragraph was that, when $q$ is even, and when $\mathcal{A}$ does not contain $P_{i}$ and $P_{j}$, then $\mathcal{A}$ contains $\bar{\Sigma}_{i}$ and $\bar{\Sigma}_{j}$ where $\Sigma_{i}$ and $\Sigma_{j}$ are respectively an $i$ - and a $j$-transversal of $\Sigma$. Notice that $\Sigma_{i}$ and $\Sigma_{j}$ may overlap.

To sum up so far:

Lemma 4.2. Under the hypotheses of Lemma 4.1, if $\mathcal{A}$ is a minimal cover of $G$, and if two point stabilisers, say $P_{i}$ and $P_{j}$, are not in $\mathcal{A}$, then:

(a) if $q$ is odd, $G=(P) \mathrm{GL}_{2}(q)$ and

$$
\Sigma \cup \Pi_{i j} \cup\{M\} \cup\left\{P_{i} \cap P_{j}\right\} \preceq \mathcal{A} ;
$$

(b) and if $q$ is even,

$$
\bar{\Sigma}_{i j} \cup\left(\Sigma \backslash \Sigma_{i j}\right) \cup \Pi_{i j} \cup\left\{P_{i} \cap P_{j}\right\} \preceq \mathcal{A}
$$

where $\Sigma_{i j}$ is the union of an $i$-transversal and a $j$-transversal of $\Sigma$.

Analogously, when just one point stabiliser is absent from a minimal cover, we have the following lemma.

LEMma 4.3. Under the hypotheses of Lemma 4.1, if $\mathcal{A}$ is a minimal cover of $G$ lacking just one point stabiliser $P_{i}$ then:

(a) if $q$ is odd, $\Sigma \cup \Pi_{i} \cup\left\{N_{i} Z\right\} \preceq \mathcal{A}$;

(b) and if $q$ is even, $\bar{\Sigma}_{i} \cup\left(\Sigma \backslash \Sigma_{i}\right) \cup \Pi_{i} \preceq \mathcal{A}$ where $\Sigma_{i}$ is an $i$-transversl of $\Sigma$.

Proof: We have $\Sigma \cup \Pi_{i} \preceq \mathcal{A}$. When $q$ is odd $N_{i} Z$ intersects trivially every Singer normaliser and every $P_{j}(j \neq i)$, using a familiar argument. This proves the first statement. The second statement uses the argument given in the preamble to Lemma 4.2.

We are now in a position to describe the minimised covers of $G$.

THEOREM 4.4. Let $q \geqslant 7$ and $G=(\mathrm{P}) \mathrm{GL}_{2}(q)$, or $q \geqslant 4$ but $q \neq 5,7,9,11$ and $G=(P) S L_{2}(q)$. The minimal members of $\Gamma_{G}$ are precisely:

1. $q$ odd

(a) $\Sigma \cup \Pi_{i j} \cup\{M\} \cup\left\{P_{i} \cap P_{j}\right\}(1 \leqslant i<j \leqslant q+1)\left(G=(\mathrm{P}) \mathrm{GL}_{2}(q)\right.$ only);

(b) $\Sigma \cup \Pi_{i} \cup\left\{N_{i} Z\right\}(1 \leqslant i \leqslant q+1)$.

2. $q$ even

(a) $\bar{\Sigma}_{i j} \cup\left(\Sigma \backslash \Sigma_{i j}\right) \cup \Pi_{i j} \cup\left\{P_{i} \cap P_{j}\right\}(1 \leqslant i<j \leqslant q+1)$ where $\Sigma_{i j}$ is the union of an $i$-transversal and a $j$-transversal of $\Sigma$. 
(b) $\bar{\Sigma}_{i} \cup\left(\Sigma \backslash \Sigma_{i}\right) \cup \Pi_{i}$ where $\Sigma_{i}$ is an $i$-transversal of $\Sigma$ which, for no $j \neq i$, is a $j$-transversal.

Proof: Observe first of all that the unions displayed in the theorem are covers of $G$. By Lemma 3.1 each of $1(\mathrm{a})$ and $2(\mathrm{a})$ is a cover if the elements of $\left(P_{i} \backslash P_{j}\right) \cup\left(P_{j} \backslash P_{i}\right)$ are accounted for. These are either in $\left\{N_{i} Z\right\} \cup\left\{N_{j} Z\right\}$, or have order dividing $q-1$ and do not fix both $i$ and $j$. The first type are in $M$ ( $q$ odd) or in $\bar{\Sigma}_{i j}$ ( $q$ even); and, by Maschke's Theorem, the elements of the second type fix a point other than $i, j$ and so are picked up by the members of $\Pi_{i j}$. A similar argument shows that the unions 1 (b) and 2(b) are covers for $G$.

Let $\mathcal{A}$ be an arbitrary minimised cover of $G$. Note that $\mathcal{A}$ lacks at least one point stabiliser. When $q$ is even this follows from Theorem 3.5. When $q$ is odd $\mathcal{A}$ would be $\Sigma \cup \Pi$ if it contained every point stabiliser; but then $\Sigma \cup \Pi_{3} \cup\left\{N_{1} Z\right\}$, for example, would be a cover properly below $\mathcal{A}$ in $\Gamma_{G}$, contradicting the assumption that $\mathcal{A}$ is minimised.

By Lemma 4.1 at most two point stabilisers do not appear in $\mathcal{A}$. If there are two, say $P_{i}$ and $P_{j}$ then, by Lemma 4.2, the unions displayed in 1(a) and 2(a) are below $\mathcal{A}$ in $\Gamma_{G}$ when $q$ is odd or even respectively. If just one point stabiliser $P_{i}$ is not in $\mathcal{A}$ then, by Lemma 4.3, the unions in 1(b) and 2(b) are below $\mathcal{A}$ in $\Gamma_{G}$, according as $q$ is odd or even. Since these unions are all covers for $G$ each is $\mathcal{A}$. Notice that in the case 2 (b) $\Sigma_{i}$ is not a $j$-transversal for $j \neq i$ since otherwise we could replace $P_{j}$ in $\mathcal{A}$ by $\mathcal{P}_{i} \cap P_{j}$ obtaining a cover of $G$ strictly below $\mathcal{A}$.

Finally the unions $\mathcal{B}$ displayed in the theorem are minimised covers. In the cases 1(a) and 2(a) this follows at once from Lemma 4.2 since a minimised cover dominated by $\mathcal{B}$ in $\Gamma_{G}$ lacks two point stabilisers. In the cases $1(b)$ and $2(b)$ if $\mathcal{B}$ is not minimised then it dominates a minimal member $\mathcal{C}$ of $\Gamma_{G}$. By Lemma 4.3, $\mathcal{C}$ lacks a point stabiliser other than $P_{i}$, say $P_{j}$. By Lemma 4.2 this means that $M \in \mathcal{C} \preceq \mathcal{B}$ in case 1(b), a contradiction, and in case $2(\mathrm{~b})$ that $\bar{\Sigma}_{j} \subseteq \mathcal{C} \preceq \mathcal{B}$, whence $\bar{\Sigma}_{j}=\bar{\Sigma}_{i}$, also a contradiction.

Corollary 4.5. If $\mathcal{A}$ is a minimal cover of $G$ then $Z$ is in every member of $\mathcal{A}$.

\section{The missing values of $\sigma(G)$}

Here we calculate $\sigma$ for the groups $\mathrm{GL}_{2}(q), \mathrm{SL}_{2}(q), \mathrm{PSL}_{2}(q)$ and $\mathrm{PGL}_{2}(q)$ missing from Theorem 3.5 .

CASE $q=2$. In this case $Z=1$, all four groups are isomorphic to $S_{3}$, and plainly

$$
\sigma(G)=4 \text {. }
$$

CASE $q=3$. Observe that $\mathrm{PGL}_{2}(3) \cong S_{4}$ and that $A_{4}$ and the three Sylow 2-subgroups constitute a minimal cover of $S_{4}$. On the other hand $\mathrm{PSL}_{2}(3) \cong A_{4}$. It is easily seen that a minimal cover for $A_{4}$ consists of its five Sylow subgroups, so

$$
\sigma\left((\mathrm{P}) \mathrm{GL}_{2}(3)\right)=4 ; \sigma\left((\mathrm{P}) \mathrm{SL}_{2}(3)\right)=5 \text {. }
$$


CASE $q=5$. Only (P)SL $2(q)$ escape Theorem 3.5. Since $\mathrm{PSL}_{2}(5) \cong \mathrm{PSL}_{2}(4)$,

$$
\sigma\left((\mathrm{P}) \mathrm{SL}_{2}(5)\right)=10 .
$$

CASE $q=7$. Again only $(\mathrm{P}) \mathrm{SL}_{2}(q)$ are missing from Theorem 3.5. Since $G=\mathrm{PSL}_{2}(7) \cong$ $\mathrm{GL}_{3}(2)$ we can regard $G$ as acting on the projective space $\mathcal{P}(2,2)$. In this action every element of $G$ either fixes a point, or lies in a Singer cycle. There are eight Singer cycles, and seven point stabilisers, so $\sigma(G) \leqslant 15$.

By Kantor [4] the normalisers of these eight Singer subgroups, Frobenius groups of order 21, are the unique maximal subgroups of $G$ containing a Singer cycle. Hence the eight Singer normalisers all occur in a minimal cover $\mathcal{A}$ of maximal subgroups of $G$.

The only other maximal subgroups of $G$ are the point and line stabilisers, all isomorphic to $S_{4}$ : see the Atlas [2]. Hence no proper subgroup of $G$ contains more than three cyclic subgroups of order 4 . There are 21 such subgroups, so there are at least 7 subgroups in $\mathcal{A}$ different from the Singer normalisers. Thus $\sigma(G) \geqslant 15$ whence

$$
\sigma\left((\mathrm{P}) \mathrm{SL}_{2}(7)\right)=15
$$

CASE $q=9$. Let $G=\mathrm{PSL}_{2}(q)$, of order 360 . The maximal subgroups of $G$ are either isomorphic to $A_{5}$, are point stabilisers which are Frobenius of order 36, or isomorphic to $S_{4}$ : see [2]. The Singer cycles here have order 5 , their normalisers have order 10, and so there are 36 in all. No more than six 5-cycles lie in a proper subgroup of $G$.

Note that $G \cong A_{6}$, so every Singer cycle is in one of the six copies of $A_{5}$ in a conjugacy class. As before $G$ is the union of Singer cycles and point stabilisers, and therefore the union of six copies of $A_{5}$ and the ten point stabilisers. Hence $\sigma(G) \leqslant 16$.

On the other hand if $\mathcal{B}:=\left\{B_{i}: 1 \leqslant i \leqslant \sigma(G)\right\}$ is a minimal cover of $G$ of maximal subgroups then at least $36 / 6=6$ members of $\mathcal{B}$ contain a 5-cycle, and these members are all isomorphic to $A_{5}$. None of these contains a 4-cycle. Let us suppose that $\sigma(G)<16$. Then there are at most nine point stabilisers in $\mathcal{B}$, say $P$ is a point stabiliser not in $\mathcal{B}$. The nine 4-cycles in $P$ are then in different members of $\mathcal{B}$ none of which are isomorphic to $A_{5}$. Hence at most six members of $\mathcal{B}$ are copies of $A_{5}$, and therefore exactly six. It follows that $\sigma(G)=15$. Let us say that $B_{i} \cong A_{5}(1 \leqslant i \leqslant 6)$ and that, for $7 \leqslant i \leqslant 15$, each $B_{i}$ contains a 4-cycle from $P$ (but, of course, contains no 5-cycle).

From now on we shall regard $G$ as $A_{6}$, and expressions such as 'point stabiliser' and 'fixed point' will refer to the natural action of $A_{6}$ on $\{1,2,3,4,5,6\}$. We denote by $Q_{i}$ the stabiliser of $i$ in this action $(1 \leqslant i \leqslant 6)$. Each $Q_{i}$ is isomorphic to $A_{5}$ and the set $\left\{Q_{i}: 1 \leqslant i \leqslant 6\right\}$ is a conjugacy class. It is well-known that there is just one other conjugacy class $\left\{R_{i}: 1 \leqslant i \leqslant 6\right\}$ of copies of $A_{5}$ in $G$ (whose actions are transitive). These two classes are distinguished by the action of their 3-cycles: those in the $Q_{i}$ fix a point, those in the $R_{i}$ fix no point. 
In $P$ there are four 3-cycles of which just two fix a point. Let us say $c_{1}$ fixes a point but $c_{2}$ fixes no point. Moreover $c_{i} \notin B_{j}(1 \leqslant i \leqslant 2,7 \leqslant j \leqslant 15)$ since $P$ is generated by every pair consisting of a 3-cycle and a 4-cycle. Hence each of $c_{1}, c_{2}$ is in some $B_{i}(1 \leqslant i \leqslant 6)$. It follows that at least one point stabiliser, say $Q_{1}$, is not in $\mathcal{B}$. Now each of the six 5-cycles in $Q_{1}$ is in some $B_{i}(1 \leqslant i \leqslant 6)$, and no two are in the same one since two together generate $Q_{1}$. Since 1 is the unique point fixed by these 5 -cycles from $Q_{1}$ it follows that no $Q_{i}$ is in $\mathcal{B}$. This contradicts that $c_{1}$ lies in some $B_{i}(1 \leqslant i \leqslant 6)$.

Hence the assumption $\sigma(G)<16$ is false. Therefore

$$
\sigma\left((\mathrm{P}) \mathrm{SL}_{2}(9)\right)=16
$$

The values of $\sigma$ for the groups $\mathrm{PSL}_{2}(7)$ and $A_{6}$ were obtained by Shieh in [7].

\section{REFERENCES}

[1] J.H.E. Cohn, 'On n-sum groups', Math. Scand. 75 (1994), 44-58.

[2] J.H. Conway, R.T.Curtis, S.P. Norton, R.A. Parker and R.A. Wilson, Atlas of finite groups (Clarendon Press, Oxford, 1985).

[3] B. Huppert, Endliche Gruppen I (Springer-Verlag, Berlin, Heidelberg, New York, 1967).

[4] W.M. Kantor, 'Linear groups containing a Singer cycle', J. Algebra 62 (1980), 232-234.

[5] B.H. Neumann, 'Groups covered by finitely many cosets', Publ. Math. Debrecen 3 (1954), 227-242.

[6] G. Scorza, 'I gruppi che possono pensarsi come somma di tre loro sottogruppi', Boll. Un. Mat. Ital. 5 (1926), 216-218.

(7] J. Shieh, Covering groups with subgroups, Grad. Dip. Thesis (The Australian National University, ACT, Australia, 1997).

[8] M.J. Tomkinson, 'Groups covered by finitely many cosets or subgroups', Comm. Alg. 15 (1987), 845-859.

[9] M.J. Tomkinson, 'Groups as the union of proper subgroups', Math. Scand. 81 (1997), 189-198.

School of Mathematical Sciences The Australian National University

Canberra, ACT 0200

Australia

Istituto di Matematica

Facoltà di Architettura

via dell'Agnolo, 14

50122 Firenze

Italia

\author{
Dipartimento di Matematica \\ viale Morgagni, 67/A \\ 50134 Firenze \\ Italia
}

